\title{
LA INNOVACIÓN COMO REQUISITO PARA LA COMPETITIVIDAD TURÍSTICA. \\ Una metodología para su descripción y análisis
}

\author{
Licenciada Hilda Puccio \\ Docente e investigadora \\ Facultad de Ciencias Aplicadas al Turismo y la Población \\ Universidad de Morón, Argentina \\ hpuccio@gmail.com \\ Licenciada Nazarena Grana \\ Docente e investigadora \\ Facultad de Ciencias Aplicadas al Turismo y la Población \\ Universidad de Morón, Argentina
}

\section{RESUMEN}

Este artículo tiene por objetivo sistematizar la experiencia llevada a cabo por la Facultad de Ciencias aplicadas al Turismo y la Población de la Universidad de Morón, Argentina, este trabajo se enmarca dentro del Plan Federal Estratégico de Turismo Sustentable de la Dirección Nacional de Calidad la cual mediante el programa de Competitividad y subprograma de Innovaciones en Destinos Turísticos piden a la universidad identificar un conjunto de indicadores de Competitividad en Destinos. Pero ante la complejidad de la tarea, y el corto plazo se decide trabajar con la Innovación como un indicador de relevancia, que permite en una primera instancia una aproximación a la realidad. Para ello y en un contexto carente de información fidedigna se opta por diseñar una metodología para relevar, describir y analizar en forma rápida y autogestionada la existencia de innovaciones en los destinos turísticos.

Palabras claves: Competitividad, innovación, tecnologías de información y comunicación descripción y análisis. 


\title{
INNOVATION AS A REQUIREMENT FOR TOURISM COMPETITIVENESS. A methodology for its description and analysis
}

\author{
Authors \\ Licenciada Hilda Puccio \\ Docente e investigadora \\ Faculty of Applied Sciences at the Tourism and Population \\ University of Morón, Argentina \\ hpuccio@gmail.com \\ Licenciada Nazarena Grana \\ Docente e investigadora \\ Faculty of Applied Sciences at the Tourism and Population \\ University of Morón, Argentina
}

\begin{abstract}
The purpose of this article is to systematize the experience carried out by the Faculty of Applied Sciences to Tourism and Population of the University of Morón, Argentina, this work is part of the Federal Strategic Plan for Sustainable Tourism of the National Quality Board which, through the Competitiveness and Innovation Tourist Destinations subprogram, asked the university to identify a set of indicators of destinations competitiveness. But due to the complexity of the task, and short term it is decided to work with Innovation as an indicator of relevance, which allows at first an approximation to reality. In order to do this, and in a context devoid of reliable information, it is decided to design a methodology to relieve, describe and analyze a in a quick and self-managed manner the existence of innovations in tourist destinations.
\end{abstract}

Key word: Competitiveness, innovation, information and communication technologies description and analysis. 


\section{INTRODUCCIÓN}

El Plan Federal Estratégico de Turismo Sustentable de la Argentina 2005- 2016 elaborado el año 2005 declara que la sustentabilidad y la competitividad atraviesan transversalmente los ejes rectores de la política Turística de la República Argentina, los cuales guiaran en conjunto las actuaciones para la puesta en marcha del Plan. En este marco la Dirección Nacional de Calidad, desarrolla el Programa de Competitividad y el Subprograma de Innovaciones en Destinos Turísticos.

Es el Plan el que define a priori a la Competitividad como "la capacidad de obtener beneficios superiores a la media del entorno de referencia y de mantenerlos de forma sostenida incluso en circunstancias cambiantes...el concepto trasciende la visión univoca basada en la productividad y el precio y aborda como propósito final la mejora de la calidad de vida de la población residente en el espacio turístico..." Este es el marco conceptual de referencia para el accionar de las distintas Direcciones que componen la Secretaria de Turismo de Nación. Precisamente es en la Dirección Nacional de Calidad, dónde se desarrolla el Programa de Competitividad y el Subprograma de Innovaciones en Destinos Turísticos a los cuales se corresponde parte el presente trabajo, en virtud del acuerdo firmado por esta institución y la Universidad de Morón.

Finalizada la colaboración entre las dos instituciones, la Universidad de Morón prosigue con el proyecto, profundizando el nivel de análisis generando nuevas perspectivas para evaluar la competitividad turística en los destinos, ya sea observando y describiendo los sistemas territoriales de innovación como así también la colecta de datos para un futuro análisis de los "recursos y las capacidades" de las empresas localizadas en los destinos.

En el destino turístico, el paisaje natural y el paisaje urbano están en las bases de las ventajas comparativas, ventajas que son preexistentes al momento de pensar y actuar para generar ventajas competitivas entendiéndose por ventajas competitivas "aquel conjunto de atributos que posee un sector, empresa o Estado que la distinguen de sus competidores permitiéndole obtener rendimientos económicos superiores al tiempo que sus productos son reconocidos por sus clientes". Tales atributos también se relacionan con el suministro eficaz y eficiente de bienes y servicios. (Hilda Puccio 2007). En la base del logro de tales atributos se halla la Innovación, entendiendo a esta como "la capacidad de generar nuevas ideas y productos y buscar nuevas oportunidades de mercado".

El producto final del Proyecto de Investigación desarrollado en el Instituto de Investigaciones Económicas aplicada al Turismo, de la Universidad de Morón, es el diseño de un modelo de análisis para evaluar la competitividad en destinos turísticos, en el cual se considera a la innovación como un factor transversal al conjunto de factores 
territoriales que lo compone.

La realidad de Latinoamérica en materia de información, expone una situación compleja y problemática en la colecta de datos en fuentes fidedignas, por ello es prioritario delinear en primera instancia los instrumentos para relevar la situación in situ de los destinos turísticos para luego sistematizar la información pertinente en vista de la conformación de un Sistema de Información Nacional.

La información que se dispone en materia de innovación en la Argentina, refiere a las innovaciones en empresas manufactureras, no registrando al presente el registro y la descripción de innovaciones en servicios y particularmente en servicios turísticos (INDEC 1998 - 2001). Con relación al uso de las TICs, la fuente de información disponible nuevamente es el INDEC, en las Encuestas en Utilización en Tecnologías de la Información en las Empresas Industriales Argentinas período (2001- 2005) que como su título lo expresa, sólo se mide en empresas manufacturera y no existe registro de las empresas de servicios. De allí surge la necesidad de contar con un instrumento, de utilización sencilla y económica para identificar y describir las innovaciones en los destinos turísticos, y lograr con ello una primera aproximación y luego una continuidad en los registros de la información sobre el tema.

Partiendo del supuesto que las innovaciones se presentan tanto con sustento tecnológico, como con sustento no tecnológico se ha buscado una unidad de análisis de acceso sencillo y que cubriera las variables "innovación tecnológica" e "innovación no tecnológica. Con relación al tipo de innovación a considerar de entre un grupo heterogéneo y numeroso que se dan en el sector servicio turístico se optó por el uso de las TICs, dada la importancia que en los últimos años ha cobrado el uso de las mismas en el crecimiento económico del sector. Por tanto las unidades de observación lo constituyen las páginas Web institucionales; en este caso de los organismos gubernamentales y de las empresas localizadas en el destino turístico de la muestra.

El instrumento consta de una guía de observación para cada dimensión (pública y privada) exhaustiva con relación a la información buscada y una matriz dónde se vuelca la información para su análisis posterior.

Finalmente se aclara que el Proyecto de Investigación se realiza desde una perspectiva territorial para el análisis de la Competitividad de un Destino Turístico (Hilda Puccio 2005), motivo por el cual no sólo se analizan la competitividad de las empresas del sector localizadas en ellos, sino que cobran importancia la dinámica territorial que generan los gobiernos locales (modernización estatal) y los Sistemas de Innovación que articulan acciones de distintos niveles jurisdiccionales (nacional, provincial y municipal) con las transferencias tecnológicas que realizan universidades e institutos de investigaciones 
tecnológicas teniendo en vista la obtención I + D + i. (Hilda Puccio 2007)

\section{MARCO CONCEPTUAL}

\section{La competitividad}

Teniendo en cuenta el concepto de competitividad definido en la letra del Plan Federal Estratégico de Turismo pero considerando que para el presente estudio es necesario operacionalizar el concepto en componentes observables en la realidad, se procede a precisar sus alcances.

Básicamente el concepto de competitividad engloba los conceptos de productividad, eficacia y rentabilidad a los cuales se anexa el de mejora en la calidad de vida. Pero es importante no perder de vista que la competitividad es un concepto económico que se puede definir como la capacidad que tiene una empresa o territorio para insertarse en nuevos mercados al tiempo que conserva las cuotas de mercado obtenidas en el pasado, en forma eficaz, alcanzando con ello un aumento de la productividad y la rentabilidad. Dicho de otra forma una empresa o sector o una nación o un territorio son competitivos cuando consiguen insertarse en nuevos mercados y conservar los ya obtenidos elevando su productividad, y su rentabilidad por que han logrado diferenciarse de sus competidores o rivales; o sea por que cuentan con un conjunto de ventajas competitivas

\section{Ventaja Competitiva}

La ventaja competitiva es una ventaja con la que cuentan las empresas o los territorios para diferenciarse de su competencia. Esta ventaja a diferencia de las ventajas comparativas no viene dada por "la naturaleza", es una ventaja que, o bien debe ser creada o inventada ex profeso, o se logra intensificando o elevando las ventajas ya existentes.

Refiriéndose al concepto de la ventaja competitiva de las naciones, Michael Porter, Director del Instituto de Estrategia y Competitividad de la Harvard Business School, observó: "La prosperidad nacional no se hereda: se crea. Para M. Porter la ventaja competitiva de un país, nación o territorio es su capacidad para incitar a las empresas (locales o extranjeras) a utilizar el país como plataforma para llevar adelante sus actividades.

En un mundo de intercambios comerciales cada vez más liberalizados, las estrategias políticas y económicas deben concentrarse en generar y mantener las ventajas competitivas dado que en la base de toda ventaja competitiva está la creatividad y el cambio, El Foro Mundial de Economía afirma que la innovación es uno de los nueve pilares de la competitividad. 


\section{Innovación}

El Manual de Frascati (OECD, 1996: 19) define la Innovación como la transformación de una idea en un producto nuevo o mejorado que es introducido en el mercado, o en un proceso de fabricación nuevo o significativamente mejorado que es utilizado en la industria o en el comercio. La novedad que define la innovación debe serlo para el mercado en el que se desenvuelve la empresa, independientemente de si este es local, regional, nacional o internacional.

El Libro Verde de la Innovación (Unión Europea 1995: 4) considera a la innovación como sinónimo de producir, asimilar y explotar con éxito una novedad, en las esferas económica y social, de forma que aporte soluciones inéditas a los problemas y permita así responder a las necesidades de las personas y de la sociedad. Los ejemplos abundan: Puesta a punto de vacunas y medicinas, mayor seguridad en el transporte (frenos ABS, bolsas inflables), comunicaciones más fáciles (telefonía móvil, videoconferencia), accesos al conocimiento más abiertos (CD Rom, multimedios), nuevos métodos de distribución (telebanco), condiciones de trabajo más llevaderas, tecnologías menos contaminantes, servicios públicos más eficaces, etc... Es importante destacar que la innovación es un proceso continuo que va desde la creación, la aplicación y la difusión de la novedad, para recomenzar con la creación.

\section{Las Innovaciones y el turismo}

En los últimos años, el sector turístico ha introducido cambios importantes con el fin de adaptarse al desarrollo y uso de las nuevas tecnologías como son los equipos informáticos o los sistemas globales de gestión, lo que ha supuesto un cambio, no sólo en los métodos de trabajo sino en la contratación de personal con un mayor nivel de cualificación. En este sentido cabe destacar que el propio desarrollo tecnológico se ha visto influenciado asimismo por la expansión de la actividad turística, la globalización de la economía, las nuevas demandas de ocio, etc., lo que ha generado y genera una mutación constante del mercado. En este contexto, las empresas se deben adaptar a las nuevas características adoptando diversas estrategias entre las cuales se hallan las denominadas nuevas tecnologías de información y comunicación (TIC). Su sigla significa "Tecnología de la Información y Comunicación". Es un término que a menudo se lo utiliza para designar "lo relativo a la informática conectada a Internet" y, especialmente, el impacto social que se produce con su uso. Las TIC agrupan un conjunto de aparatos necesarios para administrar la información y, especialmente, las computadoras y programas necesarios para convertirla, almacenarla, administrarla, transmitirla y encontrarla.

La introducción progresiva de las TIC genera cambios en la sociedad, produciendo lo que se denomina: "sociedad de la información" o "sociedad del conocimiento". Las nuevas TIC designan a la vez un conjunto de innovaciones tecnológicas pero también las herramientas que permiten una redefinición radical del funcionamiento de la sociedad. Los primeros pasos hacia una Sociedad de la Información se remontan a la invención del 
telégrafo eléctrico, pasando posteriormente por el teléfono fijo, de la radiotelefonía y, por último, de la televisión. Internet, la telecomunicación móvil y el GPS pueden considerarse como las nuevas TICs. Un buen ejemplo de la influencia de los TIC sobre la sociedad es lo que se ha dado en llamar "Gobierno Electrónico o Gobierno Virtual"

Con relación a las innovaciones en servicios, estas pueden ser tecnológicas y no tecnológicas. Por ejemplo la creación del Club Méditerranée fue un concepto muy innovador, en su momento, en el ámbito del tiempo libre, una innovación sin un contenido tecnológico intrínseco. Para su desarrollo ha utilizado en gran medida los progresos de las tecnologías informáticas y aeronáuticas por un lado y por otro ha innovado en la organización, en los procesos y en las capacidades de la empresa.

El elemento más importante dentro de las TIC en el contexto de la actividad turística es sin duda "la red". El avance de Internet, Intranet y Extranet ha transformado la comunicación y la organización de las empresas en esta última década. Las empresas pueden distribuir gran cantidad de información a costos muy bajos, al tiempo que en forma simultánea tienen la oportunidad de interactuar con sus potenciales clientes. Un claro ejemplo de "la red", para el sector turístico se visualiza en los procesos de reservas de las agencias de viaje, compañías aéreas y tour-operadores, que se han visto beneficiados con la aparición y el uso de las nuevas tecnologías de la información y comunicación.

\section{El uso de las TIC para reforzar las fuerzas competitivas}

Hoy el mundo presenta una realidad: la liberación de los mercados, con negociaciones bilaterales que evolucionan con rapidez y, la firma e implementación de acuerdos que despliegan un amplio abanico de retos y oportunidades tanto para las empresas grandes y pequeñas, como así también para los gobiernos con relación al e-government, o Gobierno Virtual como consecuencia de la implementación de regulaciones sobre seguridad en el uso de las TICS, entre otros factores. Este es un fenómeno que no se limita a una determinada región, sino que también es previsible en diversos plazos (según avances tecnológicos y capacidad económica de cada nación) su instalación y crecimiento en todas las naciones.

En los países dónde ya se viene dando este fenómeno, evolucionan aceleradamente el e-government; el $e$-commerce; el $e$ - marketing y el $e$-intelligence, a tal punto que son parte de la vida cotidiana, lo cual les permite a tales países desarrollar por cientos altos de conectividad, no sólo para el comercio sino con la sociedad civil y los gobiernos por igual en materia de: compras, pago de impuestos, pago de servicios, negociaciones, acceso a la información gubernamental, se realizan online en ocasiones con seguridad y de manera instantánea, estos avances tecnológicos permiten ahorrar dinero y pérdida de tiempo generando un incremento de la productividad, incidiendo directamente en 
la competitividad de las empresas y la capacidad operativa de los gobiernos, todo ello redunda en la satisfacción del cliente y en beneficios para la sociedad civil.

Desde la bibliografía académica se concibe como competitivo aquel destino turístico que tiene la capacidad por un lado de atraer a la demanda potencial y por otro la de motivar a su demanda real a repetir la visita, incrementando así su productividad y rentabilidad. Los destinos turísticos deberán crear nuevas ventajas competitivas para competir con sus rivales en un mercado, al que las nuevas tendencias de consumo lo muestran altamente diversificado en segmentos cada vez más diferenciados, requiriendo para ello la implementación de estrategias de diferenciación, por lo tanto a mayor diferenciación, mayor cuota de innovación es por ello que la capacidad de innovación que desarrolla un destino se constituye en un parámetro a tener en cuenta al momento de evaluar y diagnosticar la competitividad de los mismos

La afirmación "si bien son las empresas de un destino las que deben innovar para competir en el mercado, es la gestión pública del turismo, la que imprime dinamismo a los procesos de innovación para la competitividad", está indicando que en un proceso de evaluación de la competitividad en destinos turísticos es importante observar la aplicación de las Innovaciones tanto en el sector gobierno, como en el sector empresarial.

\section{DESARROLLO}

\section{Objetivos del estudio}

Diseñar y poner aprueba un instrumento de recolección de datos y sus matrices de análisis referido a la innovación como una de las variables del modelo para estudiar la Competitividad en Destinos Turísticos de Argentina.

Adaptar el modelo obtenido para ser usado como instrumento de diagnóstico tanto para el sector público como del sector privado de las comunidades locales

\section{METODOLOGÍA}

Para el presente estudio se considera a la Capacidad Innovación que desarrolla un destino turístico como parámetro para analizar la competitividad en dicho destino se analizan las entidades generadoras de innovación, en este caso gobiernos municipales y empresas hoteleras de 4 y 5 estrellas. Se limita la observación de las Innovaciones sólo a las denominadas TIC, en la modalidad informática + Internet, por lo tanto son unidades de observación son las páginas Web o sitios Web de gobiernos municipales y hoteles del destino. 
Para analizar las Innovaciones a nivel gobiernos se observan sus páginas Web evaluando parámetros de innovación, información, interacción, transacción y articulación con la ciudadanía, los datos recopilados son ingresado en una matriz Excel, la cual permite obtener información referente a la posesión o carencia de determinada innovaciones indispensable para la Competitividad turística del destino

Para analizar las Innovaciones en los hoteles se observan las páginas Web, se estudian sus productos, sus procesos, su organización, su comercialización y su medio ambiente, esto mediante observación y evaluación de sus sitios Web en términos de: Accesibilidad, Velocidad, Contenidos y Calidad según los parámetros de Miranda y Bageñil (2004), lo cual permite establecer relaciones con las innovaciones en productos, ciertos procesos, nuevas formas comercialización, e innovación en las organizaciones.

Para la tabulación y análisis de los datos obtenidos se procedió a diseñar un aserie de matrices tanto para el tema de Gobierno virtual como para comercio virtual

\section{Matriz Gobierno Virtual}

Para la creación de la matriz de evaluación de gobierno virtual en turismo el equipo se baso en el informe internacional "Reporter Global de E- Gobierno", para el presente trabajo sólo se considera la dimensión "presencia del gobierno en la red" como parámetro de Innovación. Este parámetro denota la aptitud genérica de cada gobierno para usar al Gobierno Electrónico como herramienta de información, de interacción, de transacción y de articulación con la ciudadanía. No obstante se aclara que para una mayor precisión en diagnósticos de competitividad la dimensión "presencia del gobierno en la red" deberá ser relacionado con las dimensiones "infraestructura de telecomunicaciones" y con la dimensión "capital humano" radicado en el destino turístico.

Hacia septiembre de 2003 parecieron reinstalarse en la agenda gubernamental ciertas cuestiones relativas a la modernización del estado y al gobierno electrónico, ello se manifiesta a través de las atribuciones asignadas, mediante Decreto $\mathrm{N}^{\circ} 624 / 2003$, a la Subsecretaria de la Gestión Pública dependiente de la Jefatura de Gabinete de Ministros, para conducir el desarrollo del Gobierno Electrónico e implementar políticas de modernización del estado.

Los lineamientos estratégicos del Plan Nacional de Gobierno Electrónico (PNGE) fueron aprobados por el Decreto $N^{\circ} 378 / 2005$ del 27/04/2005 (BO 28/04/2005), junto con los de los Planes Sectoriales de los Organismos de la Administración Pública Nacional (APN).Sus lineamientos centrales son los siguientes: Mejor servicio al ciudadano; Mejor gestión pública; Transparencia; Participación; Integración; Apoyo al desarrollo; Integración a la economía mundial; Reducción de costos 
Las acciones propuestas pueden ser centralizadas, descentralizadas y acciones coordinadas, para el presente trabajo se tiene en cuenta las descentralizadas por que su existencia en el ámbito de un Municipio Turístico significa una innovación por si misma en la gestión del mismo.

Las acciones descentralizadas son: a) desarrollo de planes sectoriales de Gobierno Electrónico; b) Utilización de servicios Web; c) Uso de las Tic para aumentar la transparencia; d) Desarrollo de instrumentos que promuevan la participación ciudadana; e) Creación del expediente electrónico; f) Utilización de la firma digital; y g) Seguridad informática.

La realidad nacional con relación a la implementación del Gobierno Electronico, se ve reflejada en Reporte Global de E- Gobierno (Gobierno Electrónico), en el cual Argentina aparece en el cuarto lugar de un ranking de países iberoamericanos. La información se concentra en el análisis de los países de Íbero América, al cual se ha adicionado U.S.A -como referente comparativo- por tratarse del país que exhibe el mayor valor (y la consecuente mejor posición) en el índice a escala global.

Dicho "ranking", encabezado globalmente por U.S.A, muestra que Chile (puesto 22 a escala global) encabeza la posición entre los países de Ibero América, seguido por México (puesto 30), Argentina (puesto 31), Brasil (puesto 41) y Uruguay (puesto 47). La posición de la Argentina se sustentaría simultáneamente (según el informe) en su relevante "presencia gubernamental en la red", un satisfactorio nivel en "tecnología de telecomunicaciones" y en su alto nivel de "capital humano" dentro de la región.

\section{Metodología de Evaluación para Innovaciones Gubernamentales según sus páginas webs.}

El indicador "Presencia gubernamental en la red" ("Web measure") valora la relevancia y el estadio predominante de los portales y sitios de Gobierno Electronico relativos a Turismo y gobierno de la siguiente forma a) emergente ("emerging"), b) mejorada ("enhanced"), c) interactiva ("interactive"), c) transaccional ("transactional"), y d) articulada ("networked"). Dado que la innovación a considerar en el estudio es la aplicación de la Tic que aúna informática + Internet, las categoría de análisis: a.) Información que transmite; b.) Usabilidad del Sistema; c.) Gestión online; d.) Presencia gubernamental en la red;

La información que transmite se relaciona con la variable servicios y las subvariables servicios /tipo de público. A saber: los servicios al ciudadano, al turistas, al inversionista, al desarrollador de I+D y público interno, se materializan a través de los ítems descriptos o indicadores.

La usabilidad del sistema se relaciona con la variable herramientas; la Gestión 
online se relaciona con la variable Desarrollo Operativo de la Administración Virtual y sus indicadores; el Grado de Presencia en la Red se mide en función del grado de participación política que ha logrado con los ciudadanos pudiendo ser en una primera medición emergente y mejorada o transaccional y articulada. Sus indicadores el tipo de innovación incorporada.

Cada variable obtiene un puntaje con presencia de (igual a más de 4 ítem) innovador; de (1 a 4) moderadamente innovador y 0 no innovador.

\section{Matriz Comercio Virtual}

\section{Las Tic como fuente de ventajas competitivas en empresas turística.}

Las Tics en si no tienen ningún contenido intrínseco, sólo cuando se construye el sistema de Información deseado pueden ser considerados poseedora de un valor potencial, el cual estará basado en el rango adquirido en los servicios de manejo de información que provee dicho sistema . La relación entre las Tics y la creación de valor depende esencialmente del mejoramiento de los procesos inherentes a la gestión de información, lo cual le permite a la empresa cumplir con los requisitos del cliente y alcanzar desempeño superior. Por ello si bien el sólo hecho de tener un sitio web es signo de innovación, resulta mucho más innovativo estar en procesos de mejoramiento continuo. Este se puede evaluar a través de sucesivas observaciones y mediciones en el tiempo para su posterior evaluación.

\section{Creación de valor}

El uso de Internet en las empresas permite por un lado entregar valor y por otro capturar valor.

\section{Entregar valor.}

De a cuerdo a Zeng and Reinartz (2003) Internet tiene un impacto muy diferenciado en las distintas etapas del proceso de toma de decisión del cliente. El valor agregado real para el cliente se materializa en puntos muy específicos en el proceso de compra, sin embargo la evidencia empírica muestra que las empresas se preocupan de incrementar el proceso de búsqueda en línea prestando menor atención a facilitar los procesos transacciones en línea y casi siempre ignoran que pueden ayudar al cliente a tomar la decisión de compra. Por ello Lumpki y Dess (2004) proponen un modelo que describen las actividades de Internet que agregan valor desde la perspectiva del cliente a través del aumento y facilitación de los procesos de compra. Estas actividades son búsqueda, evaluación, resolución de problemas y transacción.

a.) Búsqueda se refiere al proceso de recopilación de información y de las opciones de compra 
b.) Evaluación es el proceso de consideración de alternativas y comparación de costos, es decir brindar un servicio online que faciliten las compras comparativas proporcionando comentarios, visualización, descripción de los productos y catalogando las evaluaciones de los clientes.

c.) Resolución de problemas. Mientras la evaluación se relaciona con los productos este ítem se relaciona con los servicios que se le ofrece al cliente con el producto

d.) Transacción. Relacionada al proceso de completar la venta, incluyendo la negociación y acuerdo contractual, haciendo pagos y realizando entregas .Internet es capaz de reducir los costos y obtener velocidad de transacción.

\section{Capturar valor.}

Al generarse un valor para entregar, es posible obtener información relevante para las empresas, si se tiene el cuidado de incluir un componente "interactivo" entre firma y cliente, lo que implica que exista un flujo de información en ambos sentidos desde la firma hacia el cliente y viceversa.

\section{Metodología para evaluar innovaciones en empresas según sus páginas webs}

El diseño de un marco conceptual para la evaluación de los sitios Web debe respetar el propósito de la evaluación (como proceso) y de los resultados obtenidos (Ros 2000). El marco conceptual de referencia aquí utilizado es el de Miranda y Bageñil (2004) que adoptan como categorías de: Análisis, Accesibilidad, Velocidad, Contenidos y Calidad.

La relación entre el proceso de búsqueda con la accesibilidad, medida en su velocidad y su navegabilidad permite medir la facilidad con que el usuario puede encontrar cómodamente lo que está buscando. Y se propone la calidad del contenido como la dimensión compuesta por tres sub - dimensiones: Información (de empresas y productos), Contenido Transaccional, y Contenido de Comunicación, además para el caso de empresas turísticas se ha agregado la dimensión Contenido de Servicios.

El contenido de Información tanto comercial (productos), y no comercial (empresa) e información de contacto permite al usuario evaluar y relacionar la oferta de los productos con la información de las empresas. El contenido transaccional permite evaluar la posibilidad que tiene el sitio Web de realizar reservas y transacciones financieras en línea, el cual se relaciona con la inversión realizada y la innovación en la organización. El Contenido de servicios permite evaluar la capacidad de resolución de problemas explorando la oferta de la empresa en función a las innovaciones del servicio.

\section{Puesta a prueba del instrumento}

La metodología se puso aprueba en los Destinos Costeros, con producto estacional "sol y playa", negocio hotelería de 4 y 5 estrellas de la provincia de Buenos 
Aires, Argentina, se escogieron los destinos de Villa Gesell, Pinamar, Mar del Plata, Miramar y Necochea

Las unidades de análisis gubernamental son los sitios Web del Gobierno Municipal de Villa Gesell y del Gobierno Municipal de Pinamar, Gobierno Municipal de General Puyrredón, Ciudad de Mar del Plata; Gobierno Municipal de General Alvarado Ciudad de Miramar, y Municipio de Necochea.

Las unidades de análisis de las empresas es un universo conformado por hoteles de 4 estrellas de Villa Gesell, de Pinamar, y de Mar del Plata y de Necochea. La ciudad de Miramar no posee hoteles de 4 y 5 estrellas, no obstante se analizan la página gubernamentales de la ciudad a los efectos de establecer a posteriori las relaciones correspondientes

\section{RESULTADOS}

La figura $n^{\circ} 1$ presenta los resultados de una medición "año cero", con relación a las innovaciones tecnológicas y no tecnológicas que desarrollan las empresas hoteleras de los destinos analizados. Para su ejecución se aplicaron las matrices de Evaluación Sitios Web de Empresas Hoteleras, y de Identificación de Aplicación de Innovaciones en Empresas Hoteleras (Puccio Hilda 2007), las dimensiones analizadas: la eentrega de valor, la Captura de valor, y las Innovaciones aplicadas

Figura $\mathbf{n}^{0}$ 1: Resultados de las matrices de: Evaluación de los sitios Web pertenecientes a las empresas hoteleras, e Identificación de aplicación de innovaciones en empresas hoteleras.

\begin{tabular}{|c|c|c|c|c|c|c|c|c|c|c|}
\hline \multirow[t]{2}{*}{\begin{tabular}{|l} 
Destino \\
Hoteles
\end{tabular}} & \multicolumn{4}{|c|}{ Entrega valor } & \multicolumn{2}{|c|}{$\begin{array}{c}\text { Capturar } \\
\text { valor }\end{array}$} & \multicolumn{4}{|c|}{ Innovaciones aplicadas } \\
\hline & $1 \mathrm{E}$ & $2 E$ & $3 \mathrm{E}$ & $4 \mathrm{E}$ & $1 \mathrm{C}$ & $2 \mathrm{C}$ & $1 \mathrm{IN}$ & $2 \mathrm{IN}$ & $3 I N$ & $4 \mathrm{IN}$ \\
\hline \begin{tabular}{|l|} 
Pinamar \\
Hoteles 4* \\
$(15)$
\end{tabular} & $\begin{array}{c}83 \% \\
\text { Innovador }\end{array}$ & $\begin{array}{c}67 \% \\
\text { Innovador } \\
33 \% \text { poco } \\
\text { Innovador }\end{array}$ & $\begin{array}{c}50 \% \\
\text { Innovador }\end{array}$ & $\begin{array}{c}50 \% \\
\text { reserva } \\
\text { online }\end{array}$ & $50 \%$ & $0 \%$ & $\begin{array}{l}\text { Sin } \\
\text { registro }\end{array}$ & $\begin{array}{l}\text { Sin } \\
\text { registro }\end{array}$ & \begin{tabular}{|c|}
$83 \%$ \\
Innovador \\
\\
$17 \%$ \\
escasa \\
Innovación
\end{tabular} & $\begin{array}{l}50 \% \text { muy } \\
\text { Innovador } \\
13 \% \text { escasa } \\
\text { Innovador } \\
17 \% \text { nada }\end{array}$ \\
\hline \begin{tabular}{|l}
$\nabla$ illa \\
Gesell \\
Hoteles 4* \\
$(9)$
\end{tabular} & $\begin{array}{c}44 \% \\
\text { Innovador }\end{array}$ & $\begin{array}{c}22 \% \\
\text { Innovador }\end{array}$ & $\begin{array}{c}22 \% \\
\text { Innovador }\end{array}$ & $\begin{array}{c}33 \% \\
\text { reserva } \\
\text { online }\end{array}$ & $55 \%$ & $0 \%$ & $\begin{array}{l}\text { Sin } \\
\text { registro }\end{array}$ & $\begin{array}{l}\text { Sin } \\
\text { registro }\end{array}$ & \begin{tabular}{|c|}
$44 \%$ \\
Innovador
\end{tabular} & $\begin{array}{c}89 \% \\
\text { Innovador }\end{array}$ \\
\hline
\end{tabular}




\begin{tabular}{|c|c|c|c|c|c|c|c|c|c|c|}
\hline $\begin{array}{l}\text { Mar del } \\
\text { Plata } \\
\text { Hoteles 4* } \\
(12) \\
\text { Hoteles 5* } \\
(3)\end{array}$ & $\begin{array}{c}73 \% \text { muy } \\
\text { innovador } \\
20 \% \\
\text { innovador } \\
7 \% \text { nada } \\
\text { innovador }\end{array}$ & $\begin{array}{c}87 \% \text { muy } \\
\text { Innovador } \\
13 \% \\
\text { Innovador }\end{array}$ & $\begin{array}{c}60 \% \\
\text { Innovador }\end{array}$ & $\begin{array}{l}93 \% \\
\text { reserva } \\
\text { online }\end{array}$ & $73 \%$ & $0 \%$ & $\begin{array}{c}\text { Sin } \\
\text { registro }\end{array}$ & $\begin{array}{c}\text { Sin } \\
\text { registro }\end{array}$ & $\begin{array}{c}60 \% \\
\text { Innovador }\end{array}$ & $\begin{array}{c}93 \% \\
\text { Innovador }\end{array}$ \\
\hline $\begin{array}{l}\text { Miramar } \\
\text { Sin Hoteles } \\
4 \text { y } 5^{*}\end{array}$ & - & & & & |----- & ---- & ------ & ----- & & \\
\hline $\begin{array}{l}\text { Necochea } \\
\text { Hoteles } 4 * \\
(2)\end{array}$ & $\begin{array}{c}100 \% \\
\text { mediana } \\
\text { mente } \\
\text { Innovador }\end{array}$ & $\begin{array}{c}100 \% \\
\text { Innovador }\end{array}$ & $\begin{array}{c}50 \% \\
\text { Innovador } \\
50 \% \text { muy } \\
\text { Innovador }\end{array}$ & $\begin{array}{c}\text { Sin } \\
\text { Innovación }\end{array}$ & $0 \%$ & $0 \%$ & $\begin{array}{c}\text { Sin } \\
\text { registro }\end{array}$ & $\begin{array}{c}\text { Sin } \\
\text { registro }\end{array}$ & & $\begin{array}{l}50 \% \text { muy } \\
\text { Innovador }\end{array}$ \\
\hline
\end{tabular}

Elaboración: Autores

Los resultados obtenidos de la aplicación de la matriz de evaluación de las innovaciones gubernamentales de un destino turístico, se han volcado a la figura $\mathrm{n}^{\circ} 2$ de acuerdo a las siguientes referencias: Información que brinda el sitio como servicio (ciudadano, turistas, inversores y I+D); Usabilidad del sitio (en función de las herramientas tecnológicas); Gestión Administrativa online; y Presencia del gobierno en la red.

Figura $\mathbf{N}^{0}$ 2: Resultados de la matriz de evaluación de las innovaciones gubernamentales de un destino turístico

\begin{tabular}{|c|c|c|c|c|c|c|c|}
\hline Destino & $\begin{array}{l}\text { Información } \\
\text { Ciudadano }\end{array}$ & $\begin{array}{l}\text { Información } \\
\text { turista }\end{array}$ & $\begin{array}{l}\text { Información } \\
\text { Inversor }\end{array}$ & $\begin{array}{l}\text { Información } \\
\mathrm{I}+\mathrm{D}\end{array}$ & $\begin{array}{l}\text { Herramientas } \\
\text { tecnológicas }\end{array}$ & $\begin{array}{l}\text { Gestión } \\
\text { adm. online }\end{array}$ & $\begin{array}{l}\text { Presencia } \\
\text { gobierno en } \\
\text { la Red }\end{array}$ \\
\hline Pinamar & Buena & Buena & Satisfactoria & Satisfactoria & $\begin{array}{l}\text { Poco } \\
\text { satisfactoria }\end{array}$ & Satisfactoria & Satisfactoria \\
\hline $\begin{array}{l}\text { Villa } \\
\text { Gesell }\end{array}$ & Buena & Muy buena & $\begin{array}{l}\text { Poco } \\
\text { satisfactoria. }\end{array}$ & $\begin{array}{l}\text { Poco } \\
\text { Satisfactoria }\end{array}$ & $\begin{array}{l}\text { Poco } \\
\text { satisfactoria }\end{array}$ & Satisfactoria & $\begin{array}{l}\text { Poco } \\
\text { satisfactoria }\end{array}$ \\
\hline $\begin{array}{l}\text { Mar del } \\
\text { Plata }\end{array}$ & Buena & $\begin{array}{l}\text { Muy } \\
\text { satisfactoria }\end{array}$ & $\begin{array}{l}\text { Muy } \\
\text { satisfactoria }\end{array}$ & $\begin{array}{l}\text { Muy } \\
\text { Satisfactoria }\end{array}$ & Satisfactoria & Satisfactoria & satisfactoria \\
\hline Miramar & Inexistente & Buena & $\begin{array}{l}\text { Poco } \\
\text { satisfactoria }\end{array}$ & $\begin{array}{l}\text { Poco } \\
\text { Satisfactoria }\end{array}$ & No satisfactoria & $\begin{array}{l}\text { No } \\
\text { satisfactoria }\end{array}$ & $\begin{array}{l}\text { No } \\
\text { satisfactoria }\end{array}$ \\
\hline Necochea & Regular & Muy buena & $\begin{array}{l}\text { Poco } \\
\text { satisfactoria }\end{array}$ & $\begin{array}{l}\text { Poco } \\
\text { satisfactoria }\end{array}$ & $\begin{array}{l}\text { Poco } \\
\text { satisfactoria }\end{array}$ & $\begin{array}{l}\text { Poco } \\
\text { satisfactoria }\end{array}$ & $\begin{array}{l}\text { Poco } \\
\text { satisfactoria }\end{array}$ \\
\hline
\end{tabular}

Elaboracion: Autores 


\section{CONCLUSIONES}

Destino Pinamar, en el sector gubernamental se observan innovaciones relacionadas a las acciones descentralizadas, que lo ubican a la vanguardia en este tema, dado que mediante el uso de las Tics promueve la participación de los ciudadanos para la toma de decisión en el caso del proyecto "Revalorización de la Avenida del Mar", motivo por el cual el indicador presencia del gobierno en la red se halla en un estadio transaccional En el área de gestión online se muestra en un estadio de innovación mejorada y todavía no han logrado el trabajo colaborativo en red. También presenta innovaciones mejoradas en el uso de herramientas tecnológicas en el área de información para el ciudadano, para turistas y desarrolladores de I+D las innovaciones se hallan entre un estadio de mejorada a interactiva. En ese contexto los hoteles de 4 estrellas del destino presentan las siguientes características: más del $50 \%$ (aproximadamente) de los mismos han desarrollado las innovaciones necesarias que le permiten "entregar valor" a sus clientes y un número próximo al $40 \%$ generan valor para la empresa. Por otro lado un $50 \%$ del universo analizado también han realizado innovaciones tecnológicas (Tic) e innovaciones no tecnológicas (en organización) lo cual ha generado a su vez innovación en su inserción en los mercados.

En el sector gubernamental de Villa Gesell, se observa en su página Web que el indicador "presencia del gobierno en la red" la ubica en el estadio de las innovaciones interactiva, en el ámbito de gestión administrativa online se halla en el estadio mejorada, y en cuanto a su información como servicios su innovación es de tipo Interactiva, pero es importante resaltar con relación a información al turista es de tipo transaccional y las innovaciones en herramientas tecnológicas son satisfactorias y funcionales .

Los resultados obtenidos en la observación del universo de de hoteles de 4 estrellas del partido señala que casi el $44 \%$ de los hoteles observado presentan una innovación considerable con relación al item inserción en los mercados .Casi el 44\% con las innovaciones han permitido "capturar valor" para la empresa coincidiendo ese mismo porcentaje en adoptar innovaciones que les ha permitido "entregar valor" a los turistas, resulta llamativo que el mayor porcentaje, de casi el 90\%, de las innovaciones realizadas por estas empresas se corresponden a las innovaciones no tecnológicas (las de organización).

La página web gubernamental de Mar del Plata desarrolla una comunicación muy satisfactoria con los ciudadanos, con los turistas, los inversores y para la facilitación de la I + D, sustentan la comunicación con la incorporación de nuevas tecnologías e innovaciones en la organización, sin embargo se detectan innovaciones, ya no de excelencia en la gestión online y en la presencia gubernamental en la red, motivos suficientes que nos lleva a inducir que existe baja inversión en actualización de las herramientas de las TICs y falta de coordinación intersectorial en la administración del sector gubernamental. 
La gran mayoría de las empresas hoteleras de Mar del Plata (entre el 60\% al 97 \%) promueven innovaciones para generar valor para el cliente y en un promedio del 60 \% están capturando valor para la empresa. Se registra dos casos de innovación en procesos: un hotel con Normas ISO y otro con certificación de seguridad en la página web, no se registran innovaciones en productos, por que es la medición correspondiente al año cero, un $69 \%$ han generado innovación en la comercialización para la cual que requiere inversión en tecnología, y un 93 \% innovaron en la organización (innovación no tecnológica) inversión en recurso humano.

En la Página del Municipio de General Alvarado, se identifica el sector dedicado al turismo de la Ciudad de Miramar. La página en general muestra una buena información al turista y una inexistente información al ciudadano, poca información para el inversor y para fomentar las I +D. Sus herramientas tecnológicas no están actualizadas, lo cual incide en parte, en la gestión online no satisfactoria y su falta de presencia en la red gubernamental. En este municipio no se registran hotelería de 4 y 5 estrellas.

En la Página del Municipio de Necochea, Turismo tiene un sector específico que desarrolla muy buena comunicación con el turista, pero es casi inexistente para el ciudadano, para el inversor y para fomentar las I+ D. Escasa actualización en sus herramientas tecnológicas, que incide en una gestión online casi inexistente y deficiente presencia en la red gubernamental .Este destino no presenta hoteles de 5 estrellas y si 2 hoteles de 4 estrellas. La falta de actualización tecnológica atenta con la competitividad del $100 \%$ de las empresas hoteleras, aunque hayan logrado muy buen nivel innovación en la organización.

Dado que la Innovación es un proceso que requiere observaciones y mediciones periódicas, en el análisis de las empresas hoteleras no se ponderan las innovaciones en producto por que la presente observación se corresponde "año cero", y recién en futuras mediciones se puede evaluar las innovaciones en producto en función de los cambios realizados. 


\section{BIBLIOGRAFIA}

- Albor Garrigos, José y Márquez Patricia (2006) “Creando valor en la cadena a través de las Tic", Ponencia X Congreso de Ingeniería de la Organización, Valencia 7 y 8 de septiembre 2006.

- Araya Dusijin, Rodrigo (2004) Tres perspectivas para observar el gobierno electrónico en América Latina Puntogob. Casos y tendencias en gobierno electrónico Pág 23 Flacso-Chile; AICD-OEA.Santiago de Chile

- Argentina (2005). Plan Federal Estratégico de Turismo Sustentable "Turismo 2016", Sector - CFI, Buenos Aires.

- Arriagada Ricardo (2002) Diseño de un sistema de medición de desempeño para evaluar la gestión municipal: una propuesta metodológica. Ilpes, CEPAL, Santiago de Chile

- ONU (2001) Informe sobre Comercio Elactronico y Desarrollo.

- ONU (2004) Conferencia de las Naciones Unidas sobre Comercio y Desarrollo "Iniciativa de la UNCTAD sobre el turismo electronico" São Paulo, 13-18 de junio 2004

- Jacob Marta y Alfonso Bravo (2001) Estudio exploratorio sobre innovación en el sector turístico Balear., COTEC, España

- Jacob Marta, Tintoré Joaquín et al. (2004) Pautas de innovación en el sector turístico Balear, COTEC, España

- OECD (1997) Manual de Oslo

- Miranda, F y Bañeguil, T (2004) "Quantitative evaluation of commercial Web Sites : an empirical study of Spanish. Internacional Journal of Information Management $\mathrm{N}^{\circ} 24$ pp. $313-320$.

- OECD (2001): Innovation and Productivity in Services, Paris.

- Orfila-Sintesis, F (2003): "La gestión del cambio tecnológico en la actividadhotelera", Tesis Doctoral, Universitat de les Illes Balears.

- Porter, Michael (1999)Ventaja Competitiva. Creación y sostenimiento de un Desempeño Superior, Compañía Editorial Continental S. A. México, décima octava reimpresión.

- Wordl Economic Forum (2001) Reporter Latinoamericano de Competitividad.

- Republica de Argentina.(2005) Plan Nacional de Gobierno Electrónico (PNGE) Decreto $N^{\circ} 378 / 2005$ del 27/04/2005 (BO 28/04/2005),

- Souto Jorge ( 2006) Las encuestas de Innovación en Argentina. Tercer taller de la medición de la Sociedad de Información América Latina .Noviembre 2006

- Torrejon, Ana (2004) Ciudades Digitales Iberoamericanas, United Nations Global E-Government Readiness Report 2004

- UNTAD (2004) Iniciativa de la UNCTAD sobre el turismo electrónico, Conferencia de las Naciones Unidas sobre Comercio y Desarrollo São Paulo, 13-18 de junio 2004. 
- UNTAD (2004) Asociación para el Desarrollo: la información y el conocimiento al servicio del desarrollo.temática. Ponencia."Las TIC como instrumento de crecimiento,desarrollo y competitividad". São Paulo, 13 a 18 de junio de 2004. Tema $8 d$ ) del programa provisional

- UNTAD (2005) Informe de la reunión de expertos en TIC y turismo para el desarrollo. celebrada en el Palacio de las Naciones, Ginebra, del 30 de noviembre al 2 de diciembre de 2005

Recibido: 26/09/2008

Aprobado: 22/11/2008

Arbitrado Anónimamente. 